\title{
Surface Instability and Dislocation Nucleation in Strained Epitaxial Layers
}

\author{
O. Trushin, \\ Institute of Microelectronics and Informatics, Academy of Sciences of Russia, Yaroslavl 150007, Russia \\ E. Granato, \\ LAS, Instituto Nacional de Pesquisas Espaciais 12201-190 São José dos Campos, SP Brasil \\ S.C. Ying, J.M. Kosterlitz \\ Department of Physics, Brown University, Providence, RI 02912 USA \\ T. Ala-Nissila, and P. Salo \\ HIT and Laboratory of Physics, Helsinki University of Technology, FIN-02015 HUT, Espoo, Finland
}

Received on 23 April, 2001

\begin{abstract}
We have studied numerically the stability and defect nucleation in epitaxial layers on a substrate with lattice mismatch. Stress relaxation and energy barriers for misfit dislocation nucleation are estimated using modern methods for saddle point search based on a combination of activation with local repulsive potential and the Nudged Elastic Band method. Stress relaxation processes correspond to different transition paths from coherent to incoherent states of the epitaxial layer. Using a two-dimensional atomistic model with Lennard-Jones interacting potential, we find different equilibrium critical thickness and activation energy behavior for dislocation nucleation of epitaxial films under tensile and compressive strain. For tensile strain, the energy barrier decreases with thickness while it reaches a constant value for compressive strain.
\end{abstract}

Standard considerations of the competition between strain energy build up and strain relief due to dislocation nucleation in mismatched epitaxial films lead to an equilibrium critical thickness above which dislocations can appear spontaneously [1]. The predicted critical value however, both from continuous elastic models [2] and from models incorporating layer discreteness [3], is much smaller than the observed experimental value suggesting that the defect-free (coherent) state above the equilibrium critical thickness should be metastable. Physically, nucleation of dislocations in initially defectfree films must then proceed by an activation process that overcomes the energy barrier between the coherent and incoherent states. The nature of the instability of the coherent state however is still under current investigation $[4,5,6]$ but detailed understanding of the corresponding atomistic mechanism should be important, for example, in the fabrication of semiconducting devices $[1,2]$. The idea of strain relaxation as an activated process is supported by recent experimental results for the temperature dependence of the critical thickness [7] and has also been addressed in a kinetic semiempirical model [8]. With recent developments in computer simulation methods, it became possible to address this problem with atomistic simulations using semiempirical potentials [9, 10], such as Embedded Atom potentials and Stillinger-Weber potential. Much simpler models, using Lennard-Jones (LJ) interacting potentials in two and three dimensions, have also been used as, for example, in the molecular dynamics (MD) study of the spontaneous relaxation in a system with large film-substrate mismatch $[11,12,13]$. The importance of kinetic factors in real experiments has also been emphasized in MD simulations of the growth process [6]. However, there are important questions that have not been addressed satisfactorily, such as: the estimation of the energy barrier for misfit dislocation nucleation; atomistic mechanisms of structural transformation along the transition path; possible structure of dislocation core and the difference in kinetics of dislocation nucleation for compressive and tensile strain. In this work, we study the mechanism of dislocation nucleation by considering the relaxation processes as different transition paths from coherent to incoherent states of the epitaxial system and determine the associated energy barrier using improved methods for saddle point search in multidimensional space.

We consider a two-dimensional model of the epitaxial film and substrate where the atomic layers are confined to a plane. Interactions between atoms in the system were modeled by a generalized LJ pair potential [14]

$$
U(r)=\varepsilon\left\{\frac{n}{m-n}\left(\frac{r_{0}}{r}\right)^{m}-\frac{m}{m-n}\left(\frac{r_{0}}{r}\right)^{n}\right\}
$$

where $r$ is the interatomic distance, $\varepsilon$ the dissociation 
energy and $r_{0}$ the equilibrium distance between the atoms. This potential was initially developed for simulation of metallic systems but it is also convenient for other systems when the parameters are chosen accordingly. In the calculations, the values of these parameters were chosen to mimic the properties of copper. In this case we set $m=5, n=8$ and used a cutoff radius for the interatomic potential of $12 \AA$. The energy scale was set to $\varepsilon=3401.1 \mathrm{~K}$ to allow realistic estimates for the strength of interatomic bondings. $r_{0}$ was set to different values to represent the substrate, epitaxial film and the substrate-film interface. For the substrate $r_{0}=r_{s s}=2.5487 \AA$ and for the epitaxial film $r_{0}=r_{f f}$ was varied to give a misfit $f=\left(r_{f f}-r_{s s}\right) / r_{s s}$. For the film-substrate interaction we set $r_{0}=r_{f s}=\left(r_{f f}+r_{s s}\right) / 2$. Positive mismatch corresponds to compressive strain and negative to tensile strain when the film is coherent with the substrate. Periodic boundary conditions are imposed in the direction parallel to the film-substrate interface. Most calculations were performed on a substrate consisting of 5 layers where the bottom 2 layers are hold fixed while the top 3 layers and all layers of the film are free to move.

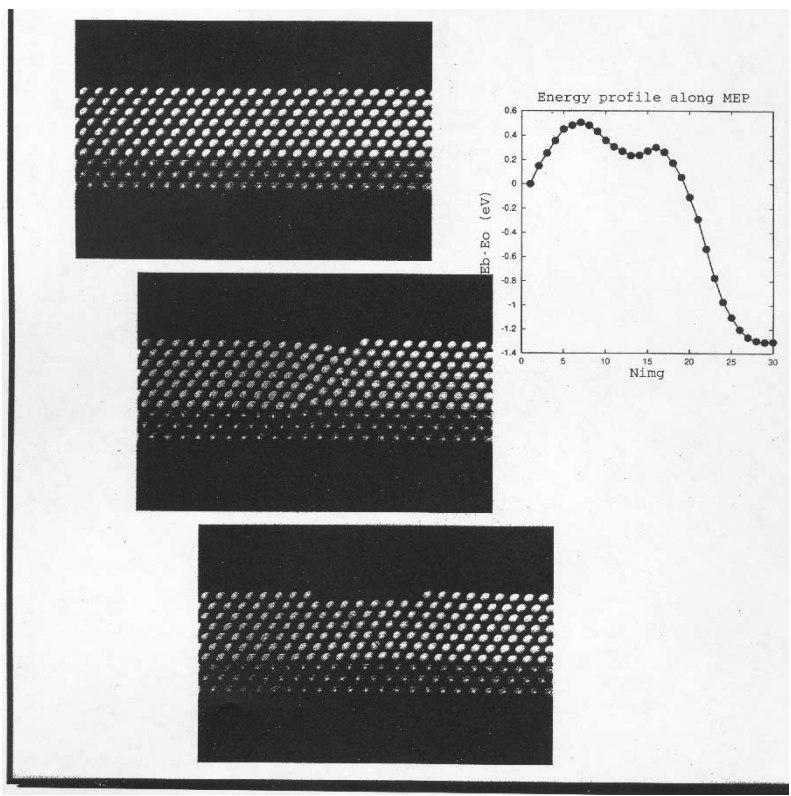

Figure 1. Particle configurations (left) in the initial epitaxial state and at different states along the minimum energy path (right), for tensile $(f=-8 \%)$ strain. $E_{b}-E_{0}$ denotes the energy change from the initial state and Nimg successive chain configurations in the Nudged Elastic Band method [15].

To study the nucleation of defects and critical thickness of this model we use a numerical technique which allows the determination of the energy profile along a transition path from the initial coherent state to a relaxed state of the epitaxial film. The technique consists of three steps. First, the initial (coherent) state is prepared by minimizing the total energy of the system through standard MD cooling [15]. (In this method, the energy is gradually minimized by setting the velocities
$V=0$ whenever $V$ and the force $F$ on a particle satisfy the condition $\vec{V} \cdot \vec{F}<0$ ). Next, the system is moved from the initial minimum to another nearest minimum energy state [16]. This is accomplished through the introduction of additional local repulsive potential of the form $U_{t o t}(r)=U(r)+A \exp \left\{-\alpha\left(r-r_{0}\right)\right\}$, where $r_{0}$ is the coordinates of the initial state at the minimum. $[16,17]$. The system is randomly displaced from the initial state in the presence of this repulsive potential to escape from the harmonic basin and arrive at the new minimum configuration again via MD cooling. Finally, with the knowledge of both the initial and final states, the minimum energy path connecting them is determined with the Nudged Elastic Band method [15]. The minimum energy path contains information for the atomic configuration at a saddle point as well as the energy barrier to escape from the initial epitaxial state. A typical result for the case of tensile strain is shown in Fig.1.

For epitaxial films above the equilibrium critical thickness, the relaxed state is expected to have a lower energy and a nonzero density of misfit dislocations which partially relieves the strain energy in the film. However, if this configuration is separated from the coherent state by a finite energy barrier the film remains coherent unless defects are nucleated allowing the system to overcome this energy barrier. This barrier could be finite even when the relaxed state with dislocations has an energy already lower than the epitaxial state. Thus the critical thickness can be much larger than the equilibrium value depending on the kinetics of defect nucleation. Preliminary results for a misfit $|f|=8 \%$ showed a large variety of relaxation processes including, single dislocation nucleation, multiple dislocations, dislocations with different core structures, dislocations nucleating on different depth in the film, which can be characterized by their different activation energies and energies of the final incoherent states. To address the problem of critical thickness we had to restrict our considerations to a small group of relaxation processes, in particular to single dislocation nucleation, with the lowest final state energy. We have performed a systematic comparison of the parameters for single dislocation nucleation processes in systems with different film thickness. We find that there is a sharp difference in the behavior of the activation barrier as function of the film thickness (number of layers) for films under compressive and tensile strain as shown in Fig. 2. In the case of compressive strain, the activation barrier increases and then saturates to some constant value whereas it decays rapidly for increasing number of layers for the tensile strain. In addition, the energy change $E_{2}-E_{1}$ from the coherent $\left(E_{1}\right)$ to the incoherent state $\left(E_{2}\right)$ also shows different behavior. The film thickness where this energy vanishes defines the equilibrium critical thickness which results smaller for compressive strain in agreement with other calculations for a similar model [6]. By slightly heating the system and performing direct MD simulation, we have also confirmed that for tensile 
strain dislocation nucleation can in fact be activated even at the lowest temperature. Spontaneous nucleation of dislocation occurs after a short time heating (100 time steps) up to $70 \mathrm{~K}$ (a negligible value of the activation energy). The difference in behavior of the energy barrier for tensile and compressive strain can be explained as resulting from the difference in the stiffness of the repulsive and attractive parts of interatomic Lennard-Jones potential. For compressive strain, displacement of the particles near the free surface cost higher energy since the interatomic potential increases sharply for separations $r<r_{o}$ whereas it decays slowly for $r>r_{o}$.
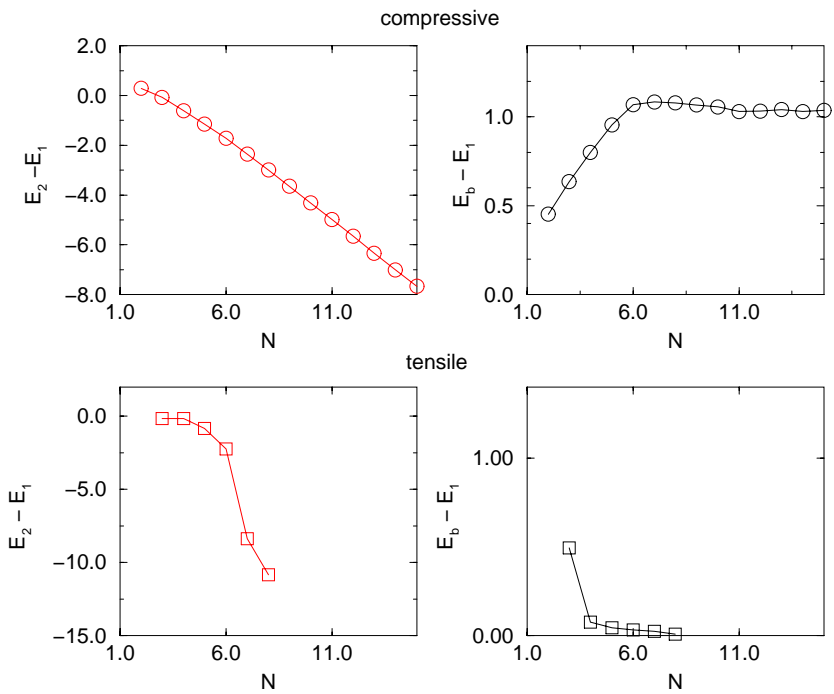

Figure 2. Relaxation energy $E_{2}-E_{1}$ (left) and energy barrier $E_{b}-E_{1}$ (right) as a function of number of layers for an epitaxial film under compressive $(f=8 \%)$ and tensile $(f=-8 \%)$ strain.

The present method can also be extended to threedimensional models with more realistic interaction potentials. Preliminary calculations for the $\mathrm{Pd} / \mathrm{Cu}$ and $\mathrm{Cu} / \mathrm{Pd}$ systems with the Embedded Atom Model potentials [18] demonstrate effectiveness of the method for systematic studies in this case as well and confirms the asymmetry of the nucleation energy with respect to the misfit as found for the simpler Lennard-Jones type potential. However, the details of the dislocation nucleation mechanism and the dependence of the barrier on the thickness in three-dimensional systems are rather different from the two-dimensional model studied here.

In summary, we have shown that by combining the Nudged Elastic Band [15] and Spherical Repulsion methods [16] it is possible to investigate in a systematic way the transition paths for misfit dislocation nucleation in epitaxial films. A large variety of processes are observed (edge dislocations, dislocations with intermixing on the film-substrate interface, dislocations with vacancy in a core and multiply nucleation). Dislocation nucleation is affected by the stiffness of interatomic potential and size of system. In the case of LJ potential, the process is sensitive to small potential cut-off radius. A nonzero activation barrier for dislocation nucleation, as found in the present model, may explain the large critical thickness and the temperature dependence often observed in experiments.

\section{Acknowledgments}

This work was supported by a joint NSF-CNPq grant, by the Russian Ministery of Science and Technology and the Academy of Finland through its Center of Excellence Program.

\section{References}

[1] J.C. Bean, Science 230, 127 (1985).

[2] C.A.B. Ball and J.H. van der Merwe, in Dislocation in Solids, F.R.N. Nabarro, Ed. (North-Holland, Amsterdam, 1983).

[3] E. Granato, J.M. Kosterlitz, and S.C. Ying, Phys. Rev. B 39, 3185 (1989).

[4] B.J. Spencer, P.W. Woorhees, S.H. Davis, Phys. Rev. Lett. 67, 3696 (1991).

[5] A.G. Cullis, A.J. Pidduck, M.T. Emeny, Phys. Rev. Lett. 75, 2368 (1995).

[6] L. Dong, J. Schnitker, R.W. Smith, and D.J. Srolovitz, J. Appl. Phys. 83, 217 (1998).

[7] J. Zou and D.J.H. Cockayne, B.F. Usher, Appl. Phys. Lett. 68673 (1996).

[8] D.C. Houghton, J. Appl. Phys. 702136 (1991).

[9] C.M. Gilmore, Phys. Rev. B 426899 (1990).

[10] M. Ichimura and J. Narayan, Phil. Mag. A, 72281 (1995).

[11] B.W. Dodson and P.A. Taylor, Appl. Phys. Lett. 49, 643 (1986).

[12] P. A. Taylor and B.W. Dodson, Phys. Rev. B 36, 1355 (1987).

[13] A.S. Nandedkar, G.R. Srinivasan, and C.S. Murthy, Phys. Rev. B 43, 7308 (1991).

[14] S. Zhen and G.J. Davies, Phys. Stat. Sol. A 78, 595 (1983).

[15] H. Jónsson, G. Mills and K. W. Jacobsen, in Classical and Quantum Dynamics in Condensed Phase Simulations, ed. by B. J. Berne et al (World Scientific, Singapore, 1998).

[16] O. Trushin, unpublished.

[17] N. Mousseau and G.T. Barkema, Phys. Rev. B 61, 1898 (2000).

[18] S.M. Foiles, M.I. Bakes, and M.S. Daw, Phys. Rev. B 33, 798 (1986). 